\title{
Cross-lingual Word Analogies using Linear Transformations between Semantic Spaces
}

\author{
Tomáš Brychcín ${ }^{1}$, Stephen Eugene Taylor ${ }^{2}$, and Lukáš Svoboda ${ }^{2}$ \\ ${ }^{1}$ NTIS - New Technologies for the Information Society, \\ Faculty of Applied Sciences, University of West Bohemia, Czech Republic \\ ${ }^{2}$ Department of Computer Science and Engineering, \\ Faculty of Applied Sciences, University of West Bohemia, Czech Republic \\ \{brychcin, taylor, svobikl\}@kiv.zcu.cz \\ http://nlp.kiv.zcu.cz
}

\begin{abstract}
We generalize the word analogy task across languages, to provide a new intrinsic evaluation method for cross-lingual semantic spaces. We experiment with six languages within different language families, including English, German, Spanish, Italian, Czech, and Croatian. State-of-the-art monolingual semantic spaces are transformed into a shared space using dictionaries of word translations. We compare several linear transformations and rank them for experiments with monolingual (no transformation), bilingual (one semantic space is transformed to another), and multilingual (all semantic spaces are transformed onto English space) versions of semantic spaces. We show that tested linear transformations preserve relationships between words (word analogies) and lead to impressive results. We achieve average accuracy of $51.1 \%, 43.1 \%$, and $38.2 \%$ for monolingual, bilingual, and multilingual semantic spaces, respectively.
\end{abstract}

\section{Introduction}

Word distributional-meaning representations have been the key in recent success in various natural language processing (NLP) tasks. The fundamental assumption (Distributional Hypothesis) is that two words are expected to be semantically similar if they occur in similar contexts (they are similarly distributed across the text). This hypothesis was formulated by Harris (1954) several decades ago. Today it is the basis of state-of-the-art distributional semantic models (Mikolov et al., 2013a; Pennington et al., 2014; Bojanowski et al., 2017).

Lately, research in distributional semantics is moving beyond monolingual representations. The research is motivated mainly by two factors: a) cross-lingual semantic representation enables reasoning about word meaning in multilingual contexts, which is useful in many applications (crosslingual information retrieval, machine translation, etc.) and b) it enables transferring of knowledge between languages, especially from resourcerich to poorly-resourced languages. Several approaches for inducing cross-lingual semantic representation (i.e., unified semantic space for different languages) have been proposed in recent years, each requiring a different form of cross-lingual supervision (Upadhyay et al., 2016). They can be roughly divided into three categories according to the level of required alignment: a) document-level alignments (Vulić and Moens, 2016), b) sentencelevel alignments (Levy et al., 2017), and c) wordlevel alignments (Mikolov et al., 2013b).

We focus on the last case, where a common approach is to train monolingual semantic spaces independently of each other and then to use bilingual dictionaries to transform semantic spaces into a unified space. Most related works rely on linear transformations (Mikolov et al., 2013b; Faruqui and Dyer, 2014; Artetxe et al., 2016) and profit from weak supervision. Vulić and Korhonen (2016) show that bilingual dictionaries with few thousand word pairs are sufficient. Such dictionaries can be easily obtained for most languages. Moreover, the mapping between semantic spaces can be easily extended to a multilingual scenario (more than two languages) (Ammar et al., 2016).

With growing attention to cross-lingual representations, it has became crucial to investigate proper evaluation schemes. Many metrics have already been proposed and they can be roughly divided into intrinsic and extrinsic evaluation metrics (Schnabel et al., 2015). In extrinsic evaluation, word representations are used as input features for a downstream task and we assess the changes in final performance. Cross-lingual applications include, e.g., sentiment analysis (Mogadala and Rettinger, 2016), document classification (Klementiev et al., 2012), or syntactic dependency parsing (Guo et al., 2015). In con- 
trast, intrinsic evaluation provides insights into the quality of representations before they are used in downstream applications. It directly tests syntactic or semantic relationships between words usually by comparison with human similarity judgments (Camacho-Collados et al., 2017).

Although neither of these metrics is perfect, there is considerable interest in evaluating semantic spaces without needing to embed them in a NLP system. Many researchers have argued that analogy is the core of cognition and have tried to address different aspects of meaning by solving word analogy problems (Turney et al., 2003; Turney, 2008; Jurgens et al., 2012). The intrinsic evaluation introduced by Mikolov et al. (2013a) gains the most attention in the last years. For example, the analogy "king is to queen as man is to woman", estimated by the vector equation king queen $\approx$ man - woman, suggests that word vectors encode information about gender. By designing appropriate analogy questions, we can implicitly test different semantic and syntactic properties of semantic spaces.

Several authors mentioned weaknesses of wordanalogy evaluation. Linzen (2016) showed that in some cases the solution is simply a nearest neighbor to the third word in the analogy question. Drozd et al. (2016) studied retrieval methods beyond vector differences to solve analogy questions and mentioned inconsistency in results. Despite these weaknesses, word analogies are still one of the most commonly used intrinsic evaluation schemes.

We are particularly concerned with intrinsic evaluations in the cross-lingual environment. Combining distributional information about words in different languages into a unified semantic space (either by mapping or by joint learning) can lose some language-specific properties. On the other hand, Faruqui and Dyer (2014) showed that canonical correlation analysis can even improve the monolingual performance on word similarity tasks by learning from multilingual contexts. Artetxe et al. (2016) have explored how crosslingual transformations affect the performance of monolingual analogies and have shown that monolingual analogy performances need not suffer from transforming semantic spaces.

In this paper, we evaluate unified semantic spaces using cross-lingual word analogies. For example, the king-queen analogy can be extended by translating the second word pair into Spanish, giving us the vector equation king - queen $\approx$ hombre - mujer. The analogy remains the same, but now it tests the ability to generalize these semantic relationships across both languages. Similarly, the analogy "walk is to walked as schwimmen (German equivalent for swim) is to schwamm (German equivalent for swam)" testifies that crosslingual word representations encode information about past tense for verbs.

To the best of our knowledge, we are the first to apply this technique of mixed language analogies. In spite of the weaknesses mentioned above, we believe it will be a valuable tool for assessing cross-lingual semantic spaces. We experiment with languages within different language families and use linear mappings to create cross-lingual semantic spaces. We extend available word-analogy corpora for English, German, Spanish, Italian, Czech, and Croatian and select only those analogy types (including both syntactic and semantic questions), which are useful among all these languages. We provide the corpus publicly available at HIDDEN-LINK. We present very promising results using transformations between any pair of six languages (43.1\% accuracy on average). Moreover, the multilingual settings (i.e., all languages are mapped onto English creating unified space for six languages) lead to only small degradation in performance compared to the bilingual case (38.2\% accuracy on average).

This paper is organized as follows. The process of learning cross-lingual word representations via linear transformations is explained in Section 2. We define the cross-lingual word analogy task and introduce the corpus for it in Section 3. The experimental results on six languages are presented and discussed in Section 4. We conclude in Section 5.

\section{Linear Transformations between Semantic Spaces}

Given a set of languages $\boldsymbol{L}$, let word $w^{a} \in \boldsymbol{V}^{a}$ denote the word in language $a \in \boldsymbol{L}$, where $\boldsymbol{V}^{a}$ is a vocabulary of that language. Let $S^{a}: \boldsymbol{V}^{a} \mapsto \mathbb{R}^{d}$ be a semantic space for language $a$, i.e., a function which projects the words $w^{a}$ into Euclidean space with dimension $d$. The meaning of the word $w^{a}$ is represented as a real-valued vector $S^{a}\left(w^{a}\right)$. We assume the same dimension $d$ for all languages ${ }^{1}$.

\footnotetext{
${ }^{1}$ Note that all described linear transformations can be easily extended to the general case, where the dimension of two
} 
This paper focuses on linear transformations between semantic spaces. A linear transformation can be expressed as

$$
S^{a \rightarrow b}\left(w^{a}\right)=S^{a}\left(w^{a}\right) \mathbf{T}^{a \rightarrow b},
$$

i.e., as a multiplication by a matrix $\mathbf{T}^{a \rightarrow b} \in \mathbb{R}^{d \times d}$.

Linear transformation can be used to perform affine transformations (e.g., rotation, reflection, translation, scaling, etc.) and other transformations (e.g., column permutation) (Nomizu and Sasaki, 1994) ${ }^{2}$. Composition of such operations is a matrix multiplication, which leads again to a matrix in $\mathbb{R}^{d \times d}$.

For estimating the transformation matrix $\mathbf{T}^{a \rightarrow b}$, we use a bilingual dictionary (set of $n$ word pairs) $\left(w^{a}, w^{b}\right) \in \boldsymbol{D}^{a \rightarrow b}$, where $\boldsymbol{D}^{a \rightarrow b} \subset \boldsymbol{V}^{a} \times \boldsymbol{V}^{b}$ and $\left|\boldsymbol{D}^{a \rightarrow b}\right|=n$. In our case, we translated the original word forms $w^{a}$ in language $a$ into language $b$ via Google translate (see Section 4). Finally, we use these $n$ aligned word pairs $\left(w^{a}, w^{b}\right)$ with their corresponding semantic vectors $\left(S^{a}\left(w^{a}\right), S^{b}\left(w^{b}\right)\right)$ to form matrices $\mathbf{X}^{a} \in \mathbb{R}^{n \times d}$ and $\mathbf{X}^{b} \in \mathbb{R}^{n \times d}$.

In the following subsections, we discuss three approaches for estimating $\mathbf{T}^{a \rightarrow b}$. The optimal transformation matrix with respect to the corresponding criteria is denoted as $\hat{\mathbf{T}}^{a \rightarrow b}$.

\subsection{Least Squares Transformation}

Following Mikolov et al. (2013b), we can estimate the matrix $\mathbf{T}^{a \rightarrow b}$ by minimizing the sum of squared residuals. The optimization problem is given by

$$
\hat{\mathbf{T}}^{a \rightarrow b}=\underset{\mathbf{T}^{a \rightarrow b}}{\arg \min }\left\|\mathbf{X}^{b}-\mathbf{X}^{a} \mathbf{T}^{a \rightarrow b}\right\|_{2}^{2}
$$

and can be solved for example by the gradient descent algorithm.

The least squares method also has an analytical solution. By taking the Moore-Penrose pseudoinverse of $\mathbf{X}^{a}$, which can be computed using sin-

${ }^{2}$ In the general case, affine transformation is the composition of two functions (a translation and a linear map) represented as $\mathbf{y}=\mathbf{A x}+\mathbf{b}$. Using so called augmented matrix (which extends the dimension by 1), we can rewrite this to $\left|\begin{array}{c}\mathbf{y} \\ 1\end{array}\right|=\left|\begin{array}{cc}\mathbf{A} & \mathbf{b} \\ 0 \ldots & 1\end{array}\right|\left|\begin{array}{c}\mathbf{x} \\ 1\end{array}\right|$, i.e., we can use only matrix multiplication (linear map). In our case, we omit this trick and use only matrix A similarly to all other prior works on linear transformations for cross-lingual NLP. Moreover, in our experiments (Section 4), we center both source and target semantic spaces towards zero so that no translation is required.
}

gular value decomposition (SVD) (Campbell and Meyer, 2009), we achieve

$$
\hat{\mathbf{T}}^{a \rightarrow b}=\left(\mathbf{X}^{a^{\top}} \mathbf{X}^{a}\right)^{-1} \mathbf{X}^{a \top} \mathbf{X}^{b} .
$$

Lazaridou et al. (2015) showed that the least squares mapping leads to increasing the hubness in the final space, because the set of vectors in $\mathbf{X}^{a} \hat{\mathbf{T}}^{a \rightarrow b}$ has lower variance than in $\mathbf{X}^{b}$ (points are on average closer to each other).

\subsection{Orthogonal Transformation}

Motivated by inconsistency among the objective functions for learning word representations (based on dot products), the least squares mapping (minimizing Euclidean distances), and word similarity evaluation (based on cosine similarities), Xing et al. (2015) argued that the transformation matrix in the least squares objective should be orthogonal. For estimating this matrix, they introduced an approximate algorithm composed of gradient descent updates and repeated applications of the SVD. Artetxe et al. (2016) then derived the analytical solution for the orthogonality constraint and showed that this transformation preserves the monolingual performance of the source space.

Orthogonal transformation is the least squares transformation subject to the constraint that the matrix $\mathbf{T}^{a \rightarrow b}$ is orthogonal ${ }^{3}$. The optimal transformation matrix is given by

$$
\hat{\mathbf{T}}^{a \rightarrow b}=\mathbf{V} \mathbf{U}^{\top},
$$

where matrices $\mathbf{V}$ and $\mathbf{U}$ are obtained using SVD of $\mathbf{X}^{b^{\top}} \mathbf{X}^{a}$ (i.e., $\mathbf{X}^{b^{\top}} \mathbf{X}^{a}=\mathbf{U} \Sigma \mathbf{V}^{\top}$ ).

\subsection{Canonical Correlation Analysis}

Canonical correlation analysis is a way of measuring the linear relationship between two multivariate variables (i.e., vectors). It finds basis vectors for each variable in the pair such that the correlation between the projections of the variables onto these basis vectors is mutually maximized.

Given the sample data $\mathbf{X}^{a}$ and $\mathbf{X}^{b}$, at the first step we look for a pair of projection vectors $\left(\mathbf{c}_{1}^{a} \in\right.$ $\mathbb{R}^{d}, \mathbf{c}_{1}^{b} \in \mathbb{R}^{d}$ ) (also called canonical directions), whose data projections $\left(\mathbf{X}^{a} \mathbf{c}_{1}^{a}, \mathbf{X}^{b} \mathbf{c}_{1}^{b}\right)$ yield the largest Pearson correlation. Once we have the best pair, we ask for the second-best pair. On either

\footnotetext{
${ }^{3}$ Matrix $\mathbf{A}$ is orthogonal if contains orthonormal rows and columns, i.e., $\mathbf{A} \mathbf{A}^{\top}=\boldsymbol{I}$. An orthogonal matrix preserves the dot product, i.e., $\mathbf{x} \cdot \mathbf{y}=(\mathbf{A x}) \cdot(\mathbf{A y})$, thus the monolingual invariance property.
} 
side of $a$ and $b$, we look for $\mathbf{c}_{2}^{a}$ and $\mathbf{c}_{2}^{b}$ in the subspaces orthogonal to the first canonical directions $\mathbf{c}_{1}^{a}$ and $\mathbf{c}_{1}^{b}$, respectively, maximizing correlation of data projections. Generally, $k$-th canonical directions are given by

$$
\left(\mathbf{c}_{k}^{a}, \mathbf{c}_{k}^{b}\right)=\underset{\mathbf{c}^{a}, \mathbf{c}^{b}}{\arg \max } \operatorname{cor}\left(\mathbf{X}^{a} \mathbf{c}^{a}, \mathbf{X}^{b} \mathbf{c}^{b}\right),
$$

where for each $1 \leq i<k,\left(\mathbf{X}^{a} \mathbf{c}^{a}\right) \cdot\left(\mathbf{X}^{a} \mathbf{c}_{i}^{a}\right)=$ 0 and $\left(\mathbf{X}^{b} \mathbf{c}^{b}\right) \cdot\left(\mathbf{X}^{b} \mathbf{c}_{i}^{b}\right)=0$. In the end of this process, we have bases of $d$ canonical directions for both sides $a$ and $b$. We can represent them as a pair of matrices $\mathbf{C}^{a} \in \mathbb{R}^{d \times d}$ and $\mathbf{C}^{b} \in \mathbb{R}^{d \times d}$ (each column corresponds to one canonical direction $\mathbf{c}_{k}^{a}$ or $\mathbf{c}_{k}^{b}$, respectively), which project $\mathbf{X}^{a}$ and $\mathbf{X}^{b}$ into a shared space. The exact algorithm for finding these bases is described in (Hardoon et al., 2004).

Faruqui and Dyer (2014) used the canonical correlation analysis for incorporating multilingual contexts into word representations, outperforming the standalone monolingual representations on several intrinsic evaluation metrics. Ammar et al. (2016) extended this work and create a multilingual semantic space for more than fifty languages. Following their approach, the final linear transformation is given by

$$
\hat{\mathbf{T}}^{a \rightarrow b}=\mathbf{C}^{a} \mathbf{C}^{b^{-1}} .
$$

\section{Cross-lingual Word Analogies}

The word analogy task consists of questions of the form: word $w_{1}$ is to $w_{2}$ as word $w_{3}$ is to $w_{4}$, where the goal is to predict $w_{4}$. Basically, the question consists of two pairs of words assuming there is the same relationship in both pairs (e.g., "Rome is to Italy in the same sense as Tokyo is to Japan").

The task was originally designed to investigate linear dependencies between words in vector space so that these questions can be answered by simple algebraic operations on corresponding word vectors (i.e., the relationship between two words is encoded as a difference of their vectors).

Similar questions can also be designed for cross-lingual cases, i.e., one pair of words is in language $a$ and second is in language $b$, e.g., "king is to queen in the same sense as Bruder (German equivalent for brother) is to Schwester (German equivalent for sister)".

More formally, we are given a word pair $\left(w_{1}^{a}, w_{2}^{a}\right)$ in language $a$ and a word $w_{3}^{b}$ in language $b$. To find the word $w_{4}^{b}$ (related to $w_{3}^{b}$ in the

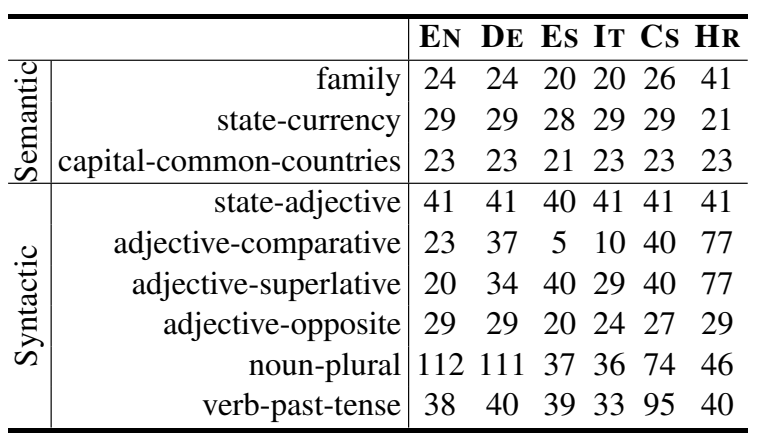

Table 1: Number of word pairs for each language and each analogy type.

same way as $w_{2}^{a}$ is related to $\left.w_{1}^{a}\right)$, we first estimate the target vector $\mathbf{v}=S^{a \rightarrow b}\left(w_{2}^{a}\right)-S^{a \rightarrow b}\left(w_{1}^{a}\right)+$ $S^{b}\left(w_{3}^{b}\right)$. Then, we go through all words $w^{b}$ in vocabulary $\boldsymbol{V}^{b}$ of language $b$ looking for the word most similar to $\mathbf{v}$ according to cosine similarity ${ }^{4}$

$$
\hat{w}_{4}^{b}=\underset{w^{b}}{\arg \max } \frac{S^{b}\left(w^{b}\right) \cdot \mathbf{v}}{\left\|S^{b}\left(w^{b}\right)\right\|_{2}\|\mathbf{v}\|_{2}} .
$$

Finally, if $\hat{w}_{4}^{b}=w_{4}^{b}$, we consider the question is answered correctly. If $a=b$, this becomes the standard monolingual word analogy task as defined in (Mikolov et al., 2013a).

We combine and extend available corpora for monolingual word analogies in English (EN) (Mikolov et al., 2013a), German (DE) (Köper et al., 2015), Spanish (Es) (Cardellino, 2016), Italian (IT) (Berardi et al., 2015), Czech (Cs) (Svoboda and Brychcín, 2016), and Croatian (HR) (Svoboda and Beliga, 2017). We consider only those analogy types, which exist across all six languages (three semantically oriented and six syntactically oriented analogy types). Table 1 shows the number of word pairs for each analogy type and each language. For all languages, questions composed of single words are taken into account (i.e., no phrases). In the following list we briefly introduce each analogy type and describe the changes and extensions we have made compared with the original corpora:

- family: Family relations based on different gender (male vs. female), e.g., son vs. daughter.

\footnotetext{
${ }^{4}$ In the monolingual case the input question words (i.e., $w_{1}, w_{2}$, and $\left.w_{3}\right)$ are discarded during the search as recommended by Mikolov et al. (2013a). In the cross-lingual case this does not make sense because $w_{1}^{a}$ and $w_{2}^{a}$ are in a different language. Thus we discard only $w_{3}^{b}$ from the search.
} 
- state-currency: Pairs representing a state and its currency, e.g., USA vs. dollar. Since this analogy type is not included in the original Czech corpus, we manually translated English word pairs.

- capital-common-countries: Word pairs consist of capital city and the corresponding state, e.g., Moscow vs. Russia.

- state-adjective: Relationship representing the state used as a noun vs. adjective, e.g., China vs. Chinese. This analogy type is not included in original Czech, Croatian, and Italian corpora. We manually translated English word pairs into these three languages.

- adjective-comparative: Adjectives in basic form and comparative form, e.g., slow vs. slower. We manually created this part for Spanish as it was not in the original corpus. Note there are very few Spanish and Italian comparatives expressed as a single word.

- adjective-superlative: Adjectives in basic form and superlative form, e.g., bad vs. worst. Similarly to adjective-comparative, we manually created this part for Spanish.

- adjective-opposite: Adjectives in basic form and negation, e.g., possible vs. impossible.

- noun-plural: Noun in basic form (lemma) and plural form, e.g., pig vs. pigs.

- verb-past-tense: Verb in infinitive and the past tense (preterite), e.g., see vs. saw.

\section{Experiments}

\subsection{Settings}

Our experiments start with building monolingual semantic spaces for each of tested languages (English, German, Spanish, Italian, Czech, and Croatian). We use character-n-gram-based skip-gram model (Bojanowski et al., 2017), which recently achieved the state-of-the-art performance in the monolingual word analogy task for several languages. For all languages except Croatian, we use word vectors pre-trained on Wikipedia ${ }^{5}$. The

\footnotetext{
${ }^{5}$ Semantic spaces for many languages trained on Wikipedia are available to download at https:// fasttext.cc. Relative sizes of Wikipedia corpora are: EN 13GB, DE 4.3GB, Es 2.5GB, It 2.3GB, Cs 0.6GB, and HR $0.2 \mathrm{~GB}$.
}

Wikipedia corpus for Croatian yields poor performance, so we combine it with web-crawled texts. We adopted the corpus $\mathrm{hrWaC}^{6}$ (Šnajder et al., 2013) and merged it with Croatian Wikipedia. The final Croatian corpus has approximately 1.3 billion tokens. We use settings recommended by Bojanowski et al. (2017), i.e., texts are lowercased, vector dimension is set to $d=300$, and character n-grams from 3 to 6 characters are used.

Bilingual dictionaries $\boldsymbol{D}^{a \rightarrow b}$ between each pair of languages $a$ and $b$, are created from the $n$ most frequent words in corpus of language $a$ and their translation into language $b$ using Google translate.

We experiment with different global postprocessing techniques for semantic spaces, which can significantly boost the final performance in word analogy task (see Section 4.3):

-c Column-wise mean centering (i.e., moving the space towards zero) is a standard step in regression analysis. Artetxe et al. (2016) showed this could lead to improving results of linear mappings.

-u Normalizing word vectors to be unit vectors guarantees that all word pairs in dictionary $\boldsymbol{D}^{a \rightarrow b}$ contribute equally to the optimization criteria of linear transformation.

-cu Column-wise mean centering followed by vector normalization.

We always apply the same post-processing for both semantic spaces $S^{a}$ and $S^{b}$ in a pair before the linear mapping. We distinguish between two types of cross-lingual semantic spaces:

B Bilingual semantic space is created by linear transformation of $S^{a}$ onto the space $S^{b}$.

M Multilingual semantic space is created by linear transformations of all $S^{a}$ except English onto the English space (i.e., unified space for all six languages).

We experiment with three techniques for linear mapping (all described in Section 2), namely, least squares transformation (LS), orthogonal transformation (OT), and canonical correlation analysis (CCA). The experiment denoted as B-OT-cu means the bilingual semantic space created by orthogonal transformation with mean centering and unit vectors. M-CCA-c means the multilingual

\footnotetext{
${ }^{6}$ Available at http: //takelab. fer.hr/data.
} 
semantic space created by canonical correlation analysis only with mean centering.

\subsection{Evaluation}

We process the questions and calculate accuracy as defined in Section 3. During the search for an answer we always browse the 300,000 most frequent words in a corresponding language. We calculate the accuracy for each analogy type separately. In prior works on monolingual word analogies, if the question or the correct answer contains an out-ofvocabulary word, it is assumed the question is answered incorrectly. The model we use in our experiments (Bojanowski et al., 2017) is able to estimate the out-of-vocabulary word representations only from the character n-grams (without context). This allows us to process all questions in the crosslingual analogy corpus.

For each analogy type we process all combinations of pairs between languages $a$ and $b$ (e.g., for the category family and the transformation from Czech to German, we have $26 \times 24=624$ questions). In the case $a=b$ (i.e., monolingual experiments), we omit the questions composed from two same pairs (e.g., for the category family in Italian, we have $20 \times 19=380$ questions). The final accuracy is an average over accuracies for individual categories. This is motivated by the fact that for each language and each analogy type, we have a different number of word pairs (see Table 1). By averaging the accuracies each analogy type contributes equally to the final score and the results are comparable across languages. In the following text, Acc@1 denotes the accuracy considering only the most similar word as a correct answer. Acc@5 assumes that the correct answer is in the list of five most similar words. All accuracies are expressed in percentages.

\subsection{Results}

Table 2 shows accuracies averaged across all combinations of pairs made of six languages. The columns represent different post-processing techniques and rows different transformations. The upper part of the table shows the monolingual experiments with original spaces without transformation (No trans.) compared with the unified multilingual space for all six languages. The orthogonal transformation provides same results as the original semantic space. Canonical correlation analysis leads to slightly lower accuracies and least squares method is worst. The most interest- ing is the lower part of the table, i.e., cross-lingual experiments, showing the average accuracies over all language pairs, but where source $a$ and target $b$ languages differ $a \neq b$. We can see that canonical correlation analysis performs best for bilingual cases, while orthogonal transformation yields better accuracies in multilingual spaces. In all cases, the mean centering followed by vector normalization led to the best results.

We chose the size of bilingual dictionaries to be $n=20,000$, because this works best among all languages (see Figure 1). This figure shows the trends for bilingual spaces with varying dictionary size. Accuracies are averaged over all source languages (monolingual spaces, i.e., where $a=b$, are not taken into account). In most cases, the accuracy decreases when $n=50,000$. We compose the bilingual dictionaries from the most frequent words. The less frequent words in dictionary may have less precise meaning representation, but all of them contribute equally to estimating the linear mapping. We believe that these less frequent words degrade the performance (i.e., more does not necessary mean better). This behavior agrees with the conclusions in (Vulić and Korhonen, 2016). Notably, we are able to achieve very promising results even with very limited dictionaries (i.e., one thousand word pairs).

Table 3 shows accuracies for all language pairs using the best settings (CCA for bilingual cases, OT for multilingual cases, $n=20,000$, and postprocessing -cu) and for both bilingual (B) and multilingual (M) case. Rows represent the source language $a$ and columns the target language $b$ (i.e., given three words $w_{1}^{a}, w_{2}^{a}$, and $w_{3}^{b}$, we look for the fourth word $w_{4}^{b}$ in column's language).

On the diagonal, we can see the monolingual results; these are the highest accuracies in each column. The highest cross-lingual accuracies are achieved by transforming onto English space (English has by far the highest monolingual accuracy), which supports our choice to use English as a intermediary for multilingual semantic spaces. We believe that English words are easier targets to hit (i.e., to find fourth word in analogy) because they are less inflected, and have fewer variations on the lemma in the same neighborhood of the semantic space. Correspondingly, the high level of inflection in Slavic languages has two consequences: the training data are diluted by the expansion of the vocabulary (both row and column effects) and 


\begin{tabular}{|c|c|c|c|c|c|c|c|c|c|}
\hline & \multirow{3}{*}{$\begin{array}{c}\text { Acc@1 } \\
49.6\end{array}$} & \multirow{3}{*}{$\begin{array}{c}\text { Acc@5 } \\
63.7\end{array}$} & \multicolumn{2}{|c|}{$-c$} & \multicolumn{2}{|c|}{$-u$} & \multicolumn{2}{|c|}{$-\mathrm{cu}$} \\
\hline & & & & Acc@1 & Acc@5 & Acc@1 & Acc@5 & Acc@1 & Acc@5 \\
\hline \multirow{4}{*}{ 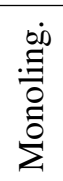 } & No trans. & & & 50.1 & 64.6 & 50.6 & 64.6 & 51.1 & 65.2 \\
\hline & M-LS & 40.2 & 55.3 & 40.3 & 55.6 & 41.3 & 56.5 & 41.3 & 56.6 \\
\hline & M-OT & 49.6 & 63.7 & 50.1 & 64.6 & 50.6 & 64.6 & 51.1 & 65.2 \\
\hline & M-CCA & 46.8 & 61.8 & 47.6 & 62.5 & 47.5 & 62.4 & 48.1 & 63.0 \\
\hline \multirow{6}{*}{ 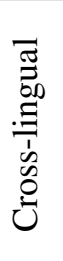 } & B-LS & 33.7 & 51.4 & 34.3 & 52.3 & 33.5 & 51.1 & 34.0 & 52.0 \\
\hline & B-OT & 40.1 & 55.9 & 40.6 & 56.6 & 40.7 & 56.5 & 41.2 & 57.3 \\
\hline & B-CCA & 42.3 & 57.5 & 42.7 & 58.2 & 42.6 & 57.8 & 43.1 & 58.5 \\
\hline & M-LS & 32.2 & 48.8 & 32.7 & 49.3 & 32.9 & 49.6 & 32.5 & 49.3 \\
\hline & M-OT & 37.3 & 53.7 & 37.6 & 54.3 & 37.8 & 54.4 & 38.2 & 55.0 \\
\hline & M-CCA & 35.3 & 52.7 & 36.2 & 53.8 & 35.5 & 52.9 & 36.0 & 53.5 \\
\hline
\end{tabular}

Table 2: The average accuracies across all combinations of language pairs for different linear transformations and post-processing techniques. The size of bilingual dictionary was set to $n=20,000$. No trans. denotes the monolingual experiments without transforming the spaces.

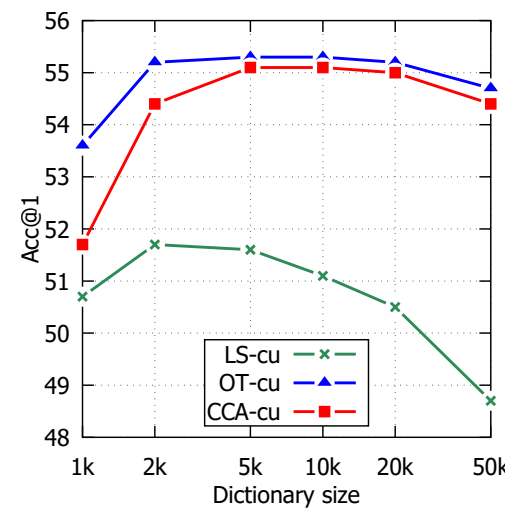

(a) EN

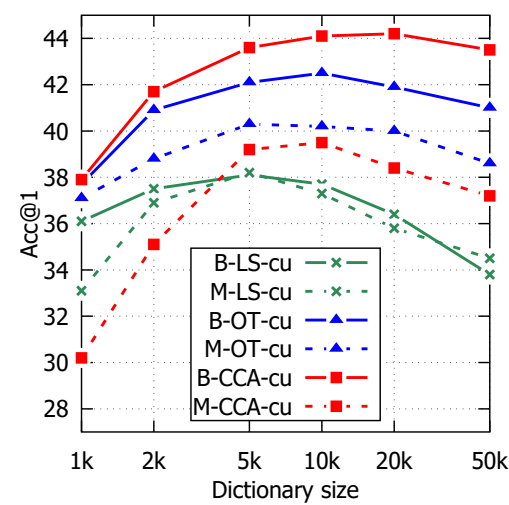

(d) IT

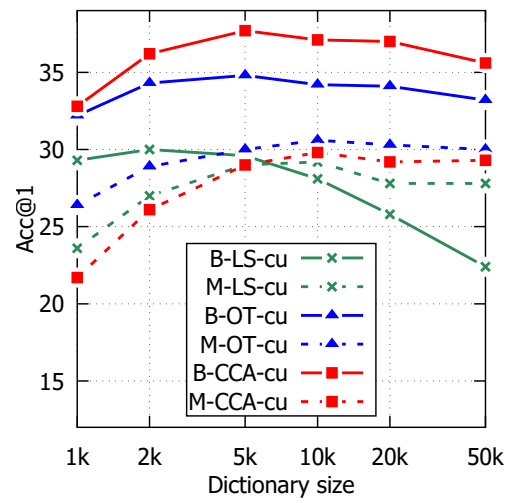

(b) $\mathrm{DE}$

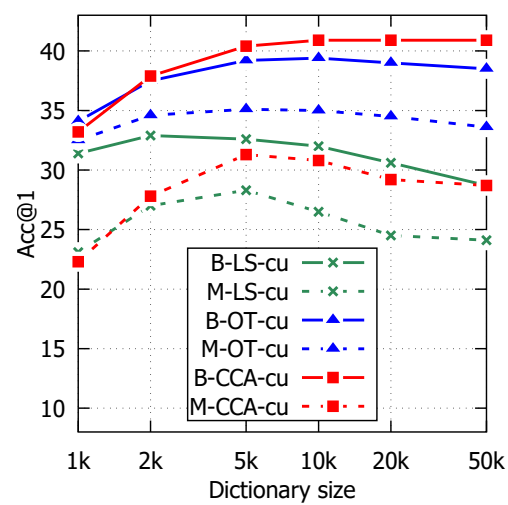

(e) Cs

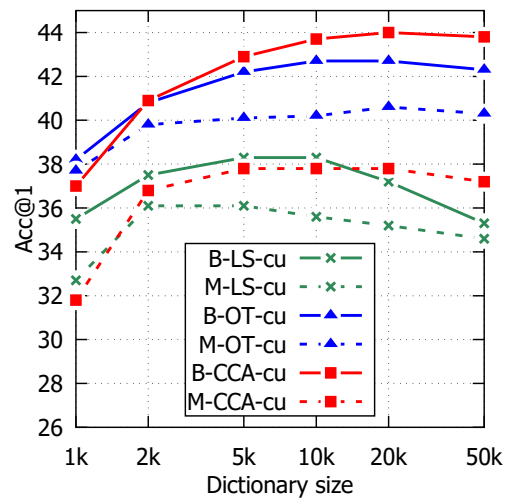

(c) Es

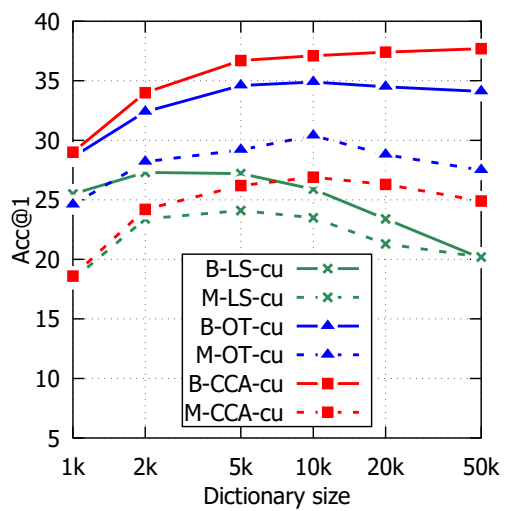

(f) $\mathrm{HR}$

Figure 1: Ranging dictionary size for all languages individually. Accuracies represent the average over all source languages except the one onto which we are transforming. Note for English (EN) both cases $\mathrm{B}$ and $\mathrm{M}$ are equal, because we transform all languages onto English to create multilingual space.

the search for the final word of the analogy has more nearby alternatives (column effect).

Table 4 shows detailed results for bilingual spaces and for each individual analogy type.
Again, rows represent the source language $a$ and columns the target language $b$. The results were achieved using B-CCA-cu transformation with dictionaries of size $n=20,000$. Each language 


\begin{tabular}{|c|c|c|c|c|c|c|c|c|c|c|c|c|}
\hline & $\begin{array}{r}\mathbf{F} \\
\text { Acc@1 }\end{array}$ & $\begin{array}{l}\mathrm{N} \\
\text { Acc@5 }\end{array}$ & $\begin{array}{r}\text { I } \\
\text { Acc@1 }\end{array}$ & $\begin{array}{l}\mathrm{E} \\
\text { Acc@5 }\end{array}$ & Acc@ & Acc@5 & Acc@1 & $\begin{array}{l}\text { T } \\
\text { Acc@5 }\end{array}$ & $\begin{array}{r}\text { ( } \\
\text { Acc@1 }\end{array}$ & Acc@5 & $\begin{array}{r}\mathbf{F} \\
\text { Acc@1 }\end{array}$ & $\begin{array}{l}\overline{\text { IR }} \\
\text { Acc@5 }\end{array}$ \\
\hline F-CCA & 63.8 & 77.0 & 41.3 & 58.7 & 45.1 & 55.8 & 44.7 & 59.6 & 43.9 & 62.5 & 41.9 & 58.7 \\
\hline M-OT & 63.8 & 77.0 & 34.5 & 54.4 & 41.4 & 54.2 & 39.8 & 56.3 & 36.3 & 56.9 & 31.5 & 52.6 \\
\hline \multirow{2}{*}{ DE $\begin{array}{c}\text { B-CCA } \\
\text { M-OT }\end{array}$} & 60.8 & 74.4 & 46.8 & 62.6 & 43.6 & 56.2 & 43.8 & 58.7 & 42.2 & 59.9 & 38.3 & 56.2 \\
\hline & 60.8 & 74.1 & 46.8 & 62.6 & 39.7 & 51.6 & 37.6 & 54.1 & 33.1 & 53.2 & 27.5 & 48.4 \\
\hline \multirow{2}{*}{ Es $\begin{array}{c}\text { B-CCA } \\
\text { M-OT }\end{array}$} & 49.2 & 63.1 & 35.9 & 50.0 & 51.3 & 62.5 & 49.7 & 63.4 & 36.9 & 51.9 & 33.6 & 49.3 \\
\hline & 49.9 & 63.7 & 29.6 & 46.3 & 51.3 & 62.5 & 46.8 & 62.4 & 32.3 & 49.1 & 26.1 & 44.5 \\
\hline \multirow{2}{*}{ IT $\begin{array}{c}\text { B-CCA } \\
\text { M-OT }\end{array}$} & 50.4 & 65.5 & 35.1 & 50.1 & 49.8 & 61.7 & 52.2 & 65.4 & 39.1 & 54.1 & 34.7 & 49.9 \\
\hline & 50.8 & 65.9 & 29.1 & 46.3 & 45.9 & 58.9 & 52.2 & 65.4 & 34.0 & 50.6 & 26.8 & 45.0 \\
\hline \multirow{2}{*}{ Cs $\begin{array}{c}\text { B-CCA } \\
\text { M-OT }\end{array}$} & 58.9 & 73.6 & 36.4 & 54.3 & 40.7 & 54.4 & 43.1 & 58.9 & 50.0 & 66.1 & 38.4 & 55.6 \\
\hline & 58.0 & 73.3 & 31.1 & 49.9 & 37.6 & 51.9 & 38.5 & 55.9 & 50.0 & 66.1 & 31.9 & 50.3 \\
\hline \multirow{2}{*}{ Hr $\begin{array}{c}\text { B-CCA } \\
\text { M-OT }\end{array}$} & 55.8 & 72.2 & 36.0 & 54.4 & 40.5 & 54.9 & 39.6 & 56.8 & 42.3 & 58.8 & 42.4 & 57.8 \\
\hline & 56.4 & 72.3 & 27.2 & 48.4 & 38.3 & 51.8 & 37.2 & 54.6 & 36.7 & 54.0 & 42.4 & 57.8 \\
\hline
\end{tabular}

Table 3: Accuracies between all pairs of languages using both bilingual spaces with CCA and multilingual semantic spaces with OT. The size of bilingual dictionaries was set to $n=20,000$. Post-processing includes mean centering and vector normalization for all cases.

\begin{tabular}{c|cccccc}
\hline & EN & DE & ES & IT & CS & HR \\
\hline EN & 68.8 & 52.4 & 85.4 & 76.0 & 41.2 & 47.2 \\
DE & 65.5 & 48.0 & 76.3 & 66.5 & 35.9 & 40.9 \\
ES & 70.6 & 49.0 & 86.8 & 74.5 & 43.1 & 45.2 \\
IT & 65.4 & 45.6 & 81.8 & 72.9 & 39.2 & 45.2 \\
CS & 61.5 & 38.6 & 74.0 & 65.0 & 35.6 & 42.0 \\
HR & 57.4 & 33.3 & 62.8 & 60.0 & 32.6 & 37.1 \\
\hline
\end{tabular}

(a) family

\begin{tabular}{c|cccccc}
\hline & EN & DE & ES & IT & CS & HR \\
\hline EN & 91.2 & 58.7 & 90.2 & 91.8 & 86.3 & 88.0 \\
DE & 91.1 & 75.9 & 86.0 & 92.8 & 73.5 & 80.2 \\
ES & 90.5 & 71.5 & 87.4 & 94.1 & 83.8 & 83.6 \\
IT & 90.5 & 61.5 & 89.8 & 89.1 & 90.1 & 85.2 \\
CS & 88.5 & 44.6 & 86.6 & 90.4 & 92.7 & 80.2 \\
HR & 86.6 & 66.8 & 82.0 & 85.4 & 82.7 & 86.5 \\
\hline
\end{tabular}

(d) state-adjective

\begin{tabular}{c|cccccc}
\hline & EN & DE & ES & IT & CS & HR \\
\hline EN & 51.4 & 39.4 & 40.7 & 38.2 & 79.8 & 49.8 \\
DE & 49.5 & 33.5 & 42.9 & 37.9 & 78.7 & 47.4 \\
ES & 46.2 & 37.2 & 40.3 & 37.3 & 76.7 & 47.2 \\
IT & 49.6 & 38.9 & 43.3 & 38.9 & 79.2 & 47.4 \\
CS & 49.6 & 35.6 & 33.9 & 34.3 & 78.9 & 41.0 \\
HR & 46.6 & 40.2 & 41.4 & 36.4 & 77.8 & 51.9 \\
\hline
\end{tabular}

(g) adjective-opposite

\begin{tabular}{c|cccccc}
\hline & EN & DE & ES & IT & CS & HR \\
\hline EN & 11.1 & 7.4 & 3.9 & 4.4 & 2.1 & 5.3 \\
DE & 5.8 & 6.7 & 1.5 & 3.2 & 1.5 & 3.4 \\
ES & 6.5 & 3.7 & 2.8 & 3.4 & 1.8 & 1.4 \\
IT & 6.3 & 4.9 & 2.8 & 3.7 & 3.0 & 3.1 \\
CS & 3.4 & 2.7 & 1.7 & 2.5 & 1.0 & 1.6 \\
HR & 5.3 & 5.7 & 1.5 & 1.6 & 1.5 & 4.3 \\
\hline
\end{tabular}

(b) state-currency

\begin{tabular}{c|cccccc}
\hline & EN & DE & ES & IT & CS & HR \\
\hline EN & 78.5 & 55.1 & 1.7 & 10.0 & 34.5 & 31.6 \\
DE & 68.4 & 59.1 & 2.2 & 7.3 & 17.4 & 16.8 \\
ES & 34.8 & 29.2 & 25.0 & 12.0 & 4.5 & 9.6 \\
IT & 41.3 & 31.9 & 8.0 & 13.3 & 4.3 & 5.5 \\
CS & 76.4 & 49.7 & 2.0 & 15.3 & 48.4 & 33.1 \\
HR & 67.6 & 45.3 & 8.3 & 15.6 & 32.6 & 32.2 \\
\hline
\end{tabular}

(e) adjective-comparative

\begin{tabular}{c|cccccc}
\hline & EN & DE & ES & IT & CS & HR \\
\hline EN & 66.8 & 48.2 & 67.6 & 45.0 & 32.6 & 40.7 \\
DE & 66.1 & 49.0 & 65.2 & 41.8 & 33.2 & 40.4 \\
ES & 68.9 & 48.6 & 71.7 & 55.4 & 32.1 & 45.0 \\
IT & 68.6 & 48.4 & 72.5 & 52.6 & 33.3 & 42.8 \\
CS & 62.2 & 43.8 & 61.7 & 36.2 & 39.4 & 31.3 \\
HR & 66.8 & 47.6 & 63.2 & 41.8 & 32.9 & 44.2 \\
\hline
\end{tabular}

(h) noun-plural

\begin{tabular}{c|cccccc}
\hline & EN & DE & ES & IT & CS & HR \\
\hline EN & 95.3 & 81.7 & 86.7 & 86.8 & 48.8 & 53.3 \\
DE & 91.9 & 82.6 & 85.9 & 89.2 & 55.0 & 49.3 \\
ES & 93.8 & 82.8 & 83.3 & 84.5 & 54.7 & 47.8 \\
IT & 93.6 & 83.2 & 85.7 & 88.9 & 54.3 & 53.1 \\
CS & 91.1 & 77.9 & 79.5 & 80.0 & 44.9 & 43.9 \\
HR & 71.1 & 55.0 & 64.4 & 55.6 & 25.9 & 32.2 \\
\hline
\end{tabular}

(c) capital-common-countries

\begin{tabular}{c|cccccc}
\hline & EN & DE & ES & IT & CS & HR \\
\hline EN & 68.9 & 15.3 & 11.5 & 20.2 & 12.0 & 19.0 \\
DE & 63.8 & 32.9 & 12.5 & 20.1 & 15.6 & 19.8 \\
ES & 4.6 & 0.4 & 32.8 & 37.3 & 0.0 & 0.2 \\
IT & 5.9 & 0.5 & 24.9 & 62.1 & 0.1 & 0.2 \\
Cs & 54.6 & 21.8 & 4.9 & 24.3 & 28.5 & 17.3 \\
HR & 57.2 & 21.6 & 7.5 & 10.8 & 21.6 & 29.2 \\
\hline
\end{tabular}

(f) adjective-superlative

\begin{tabular}{c|cccccc}
\hline & EN & DE & ES & IT & CS & HR \\
\hline EN & 42.2 & 13.1 & 18.6 & 29.7 & 58.0 & 41.8 \\
DE & 45.1 & 33.4 & 20.3 & 35.1 & 69.0 & 46.8 \\
ES & 27.0 & 0.8 & 31.8 & 48.6 & 35.2 & 22.4 \\
IT & 32.2 & 1.4 & 39.3 & 48.7 & 48.8 & 29.8 \\
CS & 42.5 & 13.1 & 22.4 & 40.0 & 80.8 & 55.6 \\
HR & 43.6 & 8.7 & 33.1 & 48.9 & 73.4 & 63.6 \\
\hline
\end{tabular}

(i) verb-past-tense

Table 4: Accuracies (Acc@ 1) of bilingual semantic spaces using B-CCA-cu for individual analogies.

seems to have strengths and weaknesses.

Interestingly, there are analogies and languages, where bilingual pairs beat monolingual. For example in the family analogies (Table 4a), English, Spanish, and Italian have the best monolingual results. Most languages profit from having the first two words of the analogy in these languages.

There is not much to say about tables $4 \mathrm{c}, 4 \mathrm{~d}$,
$4 \mathrm{~g}$, and $4 \mathrm{~h}$; all language pairs simply produce high accuracies. On the contrary, the state-currency results (Table 4b) are uniformly poor. One might expect that analogies using the national adjective would work better, because they form a frequent collocation (e.g., Hungarian forint), but those analogies also perform poorly (for $\mathrm{EN} \rightarrow \mathrm{EN}$ we achieved $12.0 \%$ ). 
In tables $4 \mathrm{e}$ and $4 \mathrm{f}$, comparative and superlative adjectives, both Romance languages (Spanish and Italian) are the anomalies. Both languages form the comparative with an adjective clitic, and both use surrounding syntax to distinguish between comparative and superlative. This syntactic dependency is sufficient to make them outliers.

In verb-past-tense (Table 4i), German is an outlier. Monolingually it works fairly well, but it frequently misses with other languages. It turns out that the cosine similarity spread and variance is greater for the German vector offsets. For all languages except English and German, the infinitive form (the first element of the word pair) is distinctively marked. In English and German, it can be confused with other forms of the verb and with nouns. Perhaps, this problem is more evident for German, where the first words in pairs may be displaced depending on the relative frequencies of the other senses.

\section{Conclusions}

In this paper we employed linear transformations to build bilingual (two languages) and multilingual (more than two languages) semantics spaces. We experimented with six languages (namely, English, German, Spanish, Italian, Czech, and Croatian) within different language families. We extended the standard word-analogy evaluation scheme onto cross-lingual environment and prepared the corpus for it. We conclude that canonical correlation analysis is more suitable for bilingual spaces and orthogonal transformation for multilingual spaces. The most important finding is that we created a unified semantic space for all six languages, which produces very promising results on word analogy task between any pair of languages in this space (average accuracy was $38.2 \%$ compared to monolingual case $51.1 \%$ ).

\section{Acknowledgments.}

This publication was supported by the project LO1506 of the Czech Ministry of Education, Youth and Sports under the program NPU I and by university specific research project SGS-2016018 Data and Software Engineering for Advanced Applications.

\section{References}

Waleed Ammar, George Mulcaire, Yulia Tsvetkov, Guillaume Lample, Chris Dyer, and
Noah A. Smith. 2016. Massively multilingual word embeddings. CoRR abs/1602.01925. http://arxiv.org/abs/1602.01925.

Mikel Artetxe, Gorka Labaka, and Eneko Agirre. 2016. Learning principled bilingual mappings of word embeddings while preserving monolingual invariance. In Proceedings of the 2016 Conference on Empirical Methods in Natural Language Processing. Association for Computational Linguistics, Austin, Texas, pages 2289-2294. https://aclweb.org/anthology/D16-1250.

Giacomo Berardi, Andrea Esuli, and Diego Marcheggiani. 2015. Word embeddings go to Italy: A comparison of models and training datasets. In Proceedings of the 6th Italian Information Retrieval Workshop, Cagliari, Italy, May 25-26, 2015.

Piotr Bojanowski, Edouard Grave, Armand Joulin, and Tomáš Mikolov. 2017. Enriching word vectors with subword information. Transactions of the Association for Computational Linguistics 5:135-146. https://transacl.org/ojs/index.php/tacl/article/view/999.

José Camacho-Collados, Mohammad Taher Pilehvar, Nigel Collier, and Roberto Navigli. 2017. SemEval2017 task 2: Multilingual and cross-lingual semantic word similarity. In Proceedings of the 11th International Workshop on Semantic Evaluation (SemEval-2017). Association for Computational Linguistics, Vancouver, Canada, pages 15-26. http://www.aclweb.org/anthology/S17-2002.

Stephen L. Campbell and Carl D. Meyer. 2009. Generalized Inverses of Linear Transformations. Society for Industrial and Applied Mathematics. https://doi.org/10.1137/1.9780898719048.

Cristian Cardellino. 2016. Spanish Billion Words Corpus and Embeddings. http://crscardellino.me/SBWCE/.

Aleksandr Drozd, Anna Gladkova, and Satoshi Matsuoka. 2016. Word embeddings, analogies, and machine learning: Beyond king - man + woman $=$ queen. In Proceedings of COLING 2016, the 26th International Conference on Computational Linguistics: Technical Papers. The COLING 2016 Organizing Committee, Osaka, Japan, pages 35193530. http://aclweb.org/anthology/C16-1332.

Manaal Faruqui and Chris Dyer. 2014. Improving vector space word representations using multilingual correlation. In Proceedings of the 14th Conference of the European Chapter of the Association for Computational Linguistics. Association for Computational Linguistics, Gothenburg, Sweden, pages 462471. http://www.aclweb.org/anthology/E14-1049.

Jiang Guo, Wanxiang Che, David Yarowsky, Haifeng Wang, and Ting Liu. 2015. Cross-lingual dependency parsing based on distributed representations. In Proceedings of the 53rd Annual Meeting of the Association for Computational Linguistics and the 7th International Joint Conference on Natural Language Processing (Volume 
1: Long Papers). Association for Computational Linguistics, Beijing, China, pages 1234-1244. http://www.aclweb.org/anthology/P15-1119.

David R. Hardoon, Sandor R. Szedmak, and John R. Shawe-Taylor. 2004. Canonical correlation analysis: An overview with application to learning methods. Neural Computation 16(12):2639-2664. http://dx.doi.org/10.1162/0899766042321814.

Zellig Harris. 1954. Distributional structure. Word 10(23):146-162.

David Jurgens, Saif Mohammad, Peter Turney, and Keith Holyoak. 2012. Semeval-2012 task 2: Measuring degrees of relational similarity. In *SEM 2012: The First Joint Conference on Lexical and Computational Semantics - Volume 1: Proceedings of the main conference and the shared task, and Volume 2: Proceedings of the Sixth International Workshop on Semantic Evaluation (SemEval 2012). Association for Computational Linguistics, Montréal, Canada, pages 356-364. http://www.aclweb.org/anthology/S12-1047.

Alexandre Klementiev, Ivan Titov, and Binod Bhattarai. 2012. Inducing crosslingual distributed representations of words. In Proceedings of COLING 2012. The COLING 2012 Organizing Committee, Mumbai, India, pages 1459-1474. http://www.aclweb.org/anthology/C12-1089.

Maximilian Köper, Christian Scheible, and Sabine Schulte im Walde. 2015. Multilingual reliability and "semantic" structure of continuous word spaces. In Proceedings of the 11th International Conference on Computational Semantics. Association for Computational Linguistics, London, UK, pages 40-45. http://www.aclweb.org/anthology/W15-0105.

Angeliki Lazaridou, Georgiana Dinu, and Marco Baroni. 2015. Hubness and pollution: Delving into cross-space mapping for zero-shot learning. In Proceedings of the 53rd Annual Meeting of the Association for Computational Linguistics and the 7th International Joint Conference on Natural Language Processing (Volume 1: Long Papers). Association for Computational Linguistics, Beijing, China, pages 270-280. http://www.aclweb.org/anthology/P15-1027.

Omer Levy, Anders Søgaard, and Yoav Goldberg. 2017. A strong baseline for learning cross-lingual word embeddings from sentence alignments. In Proceedings of the 15th Conference of the European Chapter of the Association for Computational Linguistics: Volume 1, Long Papers. Association for Computational Linguistics, Valencia, Spain, pages 765-774. http://www.aclweb.org/anthology/E171072.

Tal Linzen. 2016. Issues in evaluating semantic spaces using word analogies. In In Proceedings of the First Workshop on Evaluating Vector Space Representations for NLP. Association for Computational Linguistics, pages 13-18.
Tomáš Mikolov, Kai Chen, Greg Corrado, and Jeffrey Dean. 2013a. Efficient estimation of word representations in vector space. CoRR abs/1301.3781. http://arxiv.org/abs/1301.3781.

Tomáš Mikolov, Quoc V. Le, and Ilya Sutskever. 2013b. Exploiting similarities among languages for machine translation. CoRR abs/1309.4168. http://arxiv.org/abs/1309.4168.

Aditya Mogadala and Achim Rettinger. 2016. Bilingual word embeddings from parallel and nonparallel corpora for cross-language text classification. In Proceedings of the 2016 Conference of the North American Chapter of the Association for Computational Linguistics: Human Language Technologies. Association for Computational Linguistics, San Diego, California, pages 692-702. http://www.aclweb.org/anthology/N16-1083.

Katsumi Nomizu and Takeshi Sasaki. 1994. Affine Differential Geometry: Geometry of Affine Immersions. Cambridge Tracts in Mathematics. Cambridge University Press. https://books.google.cz/books?id=lEUVHyjQANcC.

Jeffrey Pennington, Richard Socher, and Christopher Manning. 2014. Glove: Global vectors for word representation. In Proceedings of the 2014 Conference on Empirical Methods in Natural Language Processing (EMNLP). Association for Computational Linguistics, Doha, Qatar, pages 1532-1543. http://www.aclweb.org/anthology/D14-1162.

Tobias Schnabel, Igor Labutov, David Mimno, and Thorsten Joachims. 2015. Evaluation methods for unsupervised word embeddings. In Proceedings of the 2015 Conference on Empirical Methods in Natural Language Processing. Association for Computational Linguistics, Lisbon, Portugal, pages 298-307. http://aclweb.org/anthology/D15-1036.

Lukáš Svoboda and Slobodan Beliga. 2017. Evaluation of Croatian word embeddings. CoRR abs/1711.01804. http://arxiv.org/abs/1711.01804.

Lukáš Svoboda and Tomáš Brychcín. 2016. New word analogy corpus for exploring embeddings of Czech words. CoRR abs/1608.00789. http://arxiv.org/abs/1608.00789.

Peter D. Turney. 2008. The latent relation mapping engine: Algorithm and experiments. Journal of Artificial Intelligence Research 33:615-655. https://doi.org/10.1613/jair.2693.

Peter D. Turney, Michael L. Littman, and Jeffrey Bighamand Victor Shnayder. 2003. Combining independent modules to solve multiple-choice synonym and analogy problems. In In Proceedings of the International Conference on Recent Advances in Natural Language Processing. pages 482-489.

Shyam Upadhyay, Manaal Faruqui, Chris Dyer, and Dan Roth. 2016. Cross-lingual models of word 
embeddings: An empirical comparison. In Proceedings of the 54th Annual Meeting of the Association for Computational Linguistics (Volume 1: Long Papers). Association for Computational Linguistics, Berlin, Germany, pages 1661-1670. http://www.aclweb.org/anthology/P16-1157.

Jan Šnajder, Sebastian Padó, and Željko Agić. 2013. Building and evaluating a distributional memory for Croatian. In Proceedings of the 51st Annual Meeting of the Association for Computational Linguistics (Volume 2: Short Papers). Association for Computational Linguistics, Sofia, Bulgaria, pages 784-789. http://www.aclweb.org/anthology/P13-2137.

Ivan Vulić and Anna Korhonen. 2016. On the role of seed lexicons in learning bilingual word embeddings. In Proceedings of the 54th Annual Meeting of the Association for Computational Linguistics (Volume 1: Long Papers). Association for Computational Linguistics, Berlin, Germany, pages 247-257. http://www.aclweb.org/anthology/P16-1024.

Ivan Vulić and Marie-Francine Moens. 2016. Bilingual distributed word representations from documentaligned comparable data. Journal of Artificial Intelligence Research 55:953-994.

Chao Xing, Dong Wang, Chao Liu, and Yiye Lin. 2015. Normalized word embedding and orthogonal transform for bilingual word translation. In Proceedings of the 2015 Conference of the North American Chapter of the Association for Computational Linguistics: Human Language Technologies. Association for Computational Linguistics, Denver, Colorado, pages 1006-1011. http://www.aclweb.org/anthology/N15-1104. 